Pacific Journal of Mathematic 


\title{
ON STARSHAPED SETS AND HELLY-TYPE THEOREMS
}

\author{
John D. Baildon AND Ruth Silverman
}

\begin{abstract}
Suppose an ordered pair of sets $(S, K)$ in a linear topological space is of Helly type $(n+1, n)$, i.e., for every $n+1$ distinct points in $S$ there is a point in $K$ which sees at least $n$ of them via $S$. Then if $S$ is closed, $K$ compact, and $n \geqq 3$, the nontrivial visibility sets in $K$ are pairwise nondisjoint. Sufficient conditions are obtained for $S$ to be starshaped.
\end{abstract}

Let $S$ be a subset of a linear topological space $L$. For points $x, y$ in $S$, we say $x$ sees $y$ via $S$ if and only if the segment $[x, y]$ lies in $S$. Further, the set $S$ is said to be starshaped if and only if there is some point $p$ in $S$ such that, for every $x$ in $S, p$ sees $x$ via $S$.

If $S$ and $K$ are subsets of $L$, with every point $x$ in $S$ is associated its visibility set $K(x)$, the set of all points of $K$ which $x$ sees via $S$.

We shall say $(S, K)$ has Helly-type $(s, r)$, where $r$ and $s$ are positive integers, $r \leqq s$, if for every $s$ distinct points in $S$ there is a point on $K$ seeing at least $r$ of them via $S$. Clearly, if $(S, K)$ has Helly-type $(s, r)$, and $0 \leqq i \leqq r-1$, then $(S, K)$ has Helly-type $(s-i, r-i)$.

In this paper we obtain a solution to a problem posed by Valentine, concerning sets of Helly type which are unions of a finite number of starshaped sets [3, Prob. 6.7, p. 178], and also obtain some related results. Breen [1] has given conditions in the plane for a simply connected set to be a union of two starshaped sets. We replace simple connectedness by the following:

For $S$ and $K$ subsets of a linear topological space $L$, we shall say the ordered pair $(S, K)$ has the triangle property if the interior of every triangle having an edge on $K$ and the other edges in $S$ is itself a subset of $S$.

If $S$ is a closed subset of a linear topological space $L, K$ is a compact convex subset of $L$ of dimension $k$ and $(S, K)$ has the triangle property, then $K(x)$ is compact and convex for each $x \in S$. If $(S, K)$ is of Helly type $(r, r)$, for $r \geqq k+1$, then by Helly's theorem $\cap\{K(x): x \in S\} \neq \varnothing$, and $S$ is starshaped. However, it is possible under certain conditions to weaken the hypothesis considerably, and yet reach the same conclusion.

A collection of sets $\mathscr{K}$ is said to have "piercing number" $j$ or a $j$-partition for a positive integer $j$, if $\mathscr{K}$ can be represented as a union of $j$ collections, each with a nonvoid intersection.

The classical result on $j$-partitions is a theorem by $\mathrm{H}$. Hadwiger and H. DeBrunner [2], which for convenience we state here as Theorem 1. 
THEOREM 1. For integers $r, s$ and $n$, let $J(s, r, n)$ denote the smallest integer (if one exists) for which a $j$-partition is admitted by each family $\mathscr{K}$ of compact convex sets in $R^{n}$ which has the $(s, r)$ property, i.e., for every $s$ members of $\mathscr{K}$, some $r$ have a common point. Then $J(s, r, n)=s-r+1$ whenever $r \leqq s$ and $n r \geqq(n-1) s+(n+1)$.

REMARKS. When $j=1$ and $r=n+1$, Theorem 1 reduces to Helly's theorem.

If $S$ is a closed subset of a linear topological space, $K$ a compact convex subset of $S$ of dimension $n$, such that $(S, K)$ has the triangle property and is of Helly type $(s, r)$, then for every $x \in S, K(x)$ is compact and convex, and the collection $\{K(x): x \in S\}$ has the $(s, r)$ property.

Therefore, if $J(s, r, n)=j$, then the set $S$ can be expressed as a union of $j$ starshaped sets. However, for choices of $s, r$ and $n$ as small as $s=4$, $r=3, n=2$, it is not known whether $J(4,3,2)$ exists.

If $n=1$, then Theorem 1 implies that $J(s, r, 1)=s-r+1$ if $r \geqq 2$, so that $J(s, 2,1)=s-1$. Consequently $S$ will be the union of $s-1$ starshaped sets if $(S, K)$ has Helly-type $(s, 2)$ and $K$ is a compact line segment. Also, since $J(r+1, r, 1)=2$ for all $r \geqq 2, \quad J(3,2,1)=$ $J(4,3,1)=2$. Consequently if $(S, K)$ has Helly-type $(3,2)$ or $(4,3)$, where $K$ is a compact line segment, then $S$ is the union of two starshaped sets. Breen [1] proved this result for Helly-type $(3,2)$ without the assumption that $K(x) \neq \varnothing$ for all $x$ in $S$. We improve the $(4,3)$ case by showing $S$ will be starshaped. In fact, in Theorem 4 , we obtain the more general result that if $(S, K)$ is of Helly type $(2 k+2,2 k+1)$ in a linear topological space, and $K$ is of dimension $k$, then with a single exception $S$ is starshaped. This result improves the prediction, from $J(2 k+2,2 k+$ $1, k)=2$, that $S$ would be a union of two starshaped sets. In Theorems 2 and 3 , for $(S, K)$ of Helly type $(n+1, n)$, without restrictions on dimension, sufficient conditions are obtained for the visibility sets to be pairwise nondisjoint (2), or for $S$ to be starshaped (3).

We must first prove the following lemma.

LEMMA. Let $S$ and $K$ be a closed and a compact subset, respectively, of a linear topological space $L$. If there exist $x, w$ in $S$ such that $K(x) \cap K(w)=\varnothing$ and $p \in K(x), q \in K(w)$, then there exist $t_{0}, \tau_{0}$ in $(0,1)$ such that if $|t|<t_{0},|\tau|<\tau_{0}$, then $K(y(t)) \cap K(z(\tau))=\varnothing$, where $y(t)=$ $t p+(1-t) x$, and $z(y)=\tau q+(1-\tau) w$.

Proof. We first observe that for every $x$ in $S, K(x)$ is compact: recall $K(x)=\{p \in K \cap S \mid[p, x] \subset S\}$. Let $p$ be a limit point of $K(x)$. Select a sequence $\left\{p_{n}\right\}$ such that $p_{n} \in K(x)$ for every $n$ and $p_{n} \rightarrow p$. For each $n$, the line segment $\left[p_{n}, x\right]$ is contained in $S$. By closure of $S,[p, x] \subset S$ and by closure of $K, p \in K$. Therefore $p \in$ 
$K(x)$. So $K(x)$ is a closed subset of a compact set and consequently compact.

Since $K(w)$ and $K(x)$ are compact and disjoint, there are disjoint open neighborhoods $U, U^{\prime}$ in $L$, such that $K(x) \subset U$ and $K(w) \subset U^{\prime}$.

We wish to prove the existence of $t_{0}>0$ such that $0<t<t_{0}$ implies $K(y(t)) \subset U$. Since $t_{0}$ exists trivially if $K(x)=\{x\}$, we may assume $K(x) \neq\{x\}$.

Assume no such $t_{0}$ exists. Then we can find a sequence of real numbers $\left\{t_{n}\right\}, t_{n} \rightarrow 0$ as ${ }^{2} n \rightarrow \infty$, and a corresponding sequence of points $\left\{\alpha_{n}\right\}$ in $K \sim U$, such that for every $n, \alpha_{n} \in K\left(y\left(t_{n}\right)\right)$.

By compactness of $K \sim U$, there is a point $\alpha_{0} \in K \sim U$ and a subsequence of $\left\{\alpha_{n}\right\}$, called for convenience $\left\{a_{i}\right\}$, such that $\alpha_{i} \rightarrow \alpha_{0}$ as $i \rightarrow \infty$. Now for each $i, \alpha_{i} \in K\left(y\left(t_{i}\right)\right)$, so the line segment from $y\left(t_{i}\right)$ to $\alpha_{i}$ is in $S$. By closure of $S$, the limiting line segment from $x$ to $\alpha_{0}$ is also in $S$. Therefore $x$ sees $\alpha_{0}$, contradicting the hypothesis, since $\alpha_{0}$, not being in $U$, is clearly not in $K(x)$.

The same argument implies the existence of $\tau_{0}>0$ such that for $0<\tau<\tau_{0}, K(z(\tau)) \subset U^{\prime}$. We therefore conclude that for $t, \tau$ sufficiently small, $K(y(t)) \cap K(z(\tau))=\varnothing$.

THEOREM 2. Let $S$ and $K$ be, respectively, a closed and a compact subset of a linear topological space $L$, such that $(S, K)$ is of Helly type $(n+1, n)$ for some $n \geqq 3$. Let $\mathscr{K}=\{K(x): x \in S, K(x) \not \subset\{x\}\}$. Then $\mathscr{K}$ is pairwise nondisjoint.

Proof. Suppose $\mathscr{K}$ fails to be pairwise nondisjoint and let $K(x)$ and $K(w)$ be members of $\mathscr{K}$ such that $K(x) \cap K(w)=\varnothing$. There exist neighborhoods $U, U^{\prime}$ such that $K(x) \subset U, K(w) \subset U^{\prime}$, and $U \cap U^{\prime}=$ $\varnothing$. As in the proof of the lemma, select $p \in K(x), p \neq x, q \in K(w)$, $q \neq w$, and then $y$ on $(x, p), z$ on $(w, q)$ such that $K(y) \subset U, K(z) \subset$ $U^{\prime}$. There is no point in $K$ seeing three of the four points $x, y, w, z$. Expanding the set $\{x, y, w, z\}$ if necessary, we have a contradiction of the hypothesis of Helly type $(n+1, n)$ for all $n \geqq 3$. Therefore $\mathscr{K}$ is pairwise nondisjoint.

A special case of Theorem 2 is of sufficient interest to be stated separately.

THEOREM 3. Let $S$ and $K$ be a closed and a compact subset respectively, of a linear topological space $L$, such that $(S, K)$ is of Helly type $(n+1, n)$ for some $n \geqq 3$. Let us further assume that for some $x_{11} \in S, K\left(x_{01}\right)=\{p\}, p \neq x_{0}$. Then either $S$ is starshaped relative to $p$ or $S$ is the union of an isolated point and a set starshaped relative to $p$.

Proof. Suppose $y_{1}$ and $y_{2}$ are points in $S \sim\{p\}$, such that $K\left(y_{\imath}\right) \subset\left\{y_{1}\right\}, i=1,2$. The set $\left\{x_{0}, y_{1}, y_{2}\right\}$, suitably expanded, lacks the 
$(n+1, n)$ property, since $y_{1}$ and $y_{2}$ do not see $p$, and $x_{0}$ sees neither $y_{1}$ nor $y_{2}$. Therefore there is at most one point $y$ in $S \sim\{p\}$ such that $K(y) \subset\{y\}$.

We then have, by Theorem 2, that at most one point in $S$ does not see $p$. Furthermore, any such point must be isolated, by the closure of $S$.

REMARK. It is possible for a point $x_{0}$ to be the only point with singleton visibility set. Consider the following example: Let $S=$ $\left\{(x, y) \in R^{2} \mid y \leqq x^{2}, 0 \leqq x \leqq 1,0 \leqq y \leqq 1\right\}$, and $K=\{(1, y) \mid 0 \leqq y \leqq 1\}$. Let $x_{0}=(0,0)$. Then $K\left(x_{0}\right)=\{(1,0)\}$. It is easily seen that $(S, K)$ satisfies the hypothesis of Theorem 3 , and that $(0,0)$ is the only point with the required property.

Remark. Theorems 2 and 3 do not hold when $(S, K)$ is of Helly type (3.2). An example is shown below.

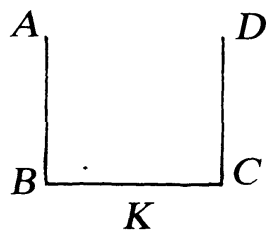

$S$ is all of broken line segment $\overline{A B C D}$, in the plane, and $K=\overline{B C}$.

REMARK. Theorems 2 and 3 trivially fail if the hypothesis lacks the condition that $K(x) \neq \varnothing$, for every $x \in S$.

REMARK. Let $S$ and $K$ be subsets of a linear topological space $L$, such that $(S, K)$ is of Helly type $(3,2)$. If there exist points $x, z \in S$ such that $K(x)=\{a\}, K(z)=\{b, c\}, a, b, c$, distinct, then $S$ is a union of three starshaped sets, since an arbitrary $w$ in $S$ sees at least one of $\{a, b, c\}$ via $S$. As we see by Breen's example [1], even with the restriction that $S$ is a closed subset of the plane and $K$ is a line segment we may need as many as three points to write $S$ as a union of starshaped sets.

THEOREM 4. Let $S$ be a closed subset of a linear topological space $L$, and let $K$ be a compact convex subset of $S$ of finite dimension $k$. Suppose $(S, K)$ has the triangle property and is of Helly type $(2 k+2,2 k+$ 1). Then $S$ is the union of a starshaped set and at most one isolated point.

Proof. Since the theorem is trivially true for $k=0$, we assume $k>0$. For arbitrary $x \in S, K(x)$ is compact, as was shown in the Lemma, and is also convex.

Suppose $K(x) \neq \varnothing$ for every $x \in S$. If, for arbitrary $\left\{x_{i}: x_{i} \in S, i=\right.$ $1,2, \cdots, k+1\}$, the set $\bigcap_{i=1}^{k+1} K\left(x_{i}\right) \neq \varnothing$, then Helly's theorem implies $\bigcap_{x \in S} K(x) \neq \varnothing$, so $S$ is starshaped. Assume $S$ is not starshaped. Then let $j$ be a minimal integer such that $\bigcap_{i=1}^{i} K\left(x_{t}\right)=\varnothing$ for some collection 
$\left\{x_{i}: x_{\imath} \in S, i=1,2, \cdots, j\right\}$. Then $j \geqq 2$ since $K(x) \neq \varnothing$ for all $x$, and $j \leqq k+1$ by assumption.

Consider $\left(S \sim\left\{x_{1}, \cdots, x_{\jmath}\right\}, K\right)$. This pair is of Helly type $(2 k+2-j$, $2 k+2-j)$ : for given an arbitrary collection of $2 k+2-j$ points from $S \sim\left\{x_{1}, \cdots, x_{j}\right\}$, augment the collection with $\left\{x_{1}, \cdots, x_{i}\right\}$, making a total of $2 k+2$ points of $S$. By hypothesis at least $2 k+1$ of these points must see a point of $K$ in common. One point from the $2 k+2$ points in $S$ must fail to see the point in $K$, in fact, a point from the set $\left\{x_{1}, \cdots, x_{j}\right\}$ since otherwise the assumption that $\bigcap_{i=1}^{\prime} K\left(x_{t}\right)=\varnothing$ would be violated. Therefore all of the $2 k+2-j$ points from $S \sim\left\{x_{1}, \cdots, x_{l}\right\}$ see the point in question.

Since $j \leqq k+1$, it follows that $2 k+2-j \geqq k+1$, so the pair $\left(S \sim\left\{x_{1}, \cdots, x_{\jmath}\right\}, K\right)$ is of Helly type $(k+1, k+1)$ as well, and consequently, by Helly's theorem $S \sim\left\{x_{1}, \cdots, x_{j}\right\}$ is starshaped. Then the closure of $S \sim\left\{x_{1}, \cdots, x_{j}\right\}$ is also starshaped. Our assumption that $S$ is not starshaped implies that there is an integer $i, 1 \leqq i \leqq j$, such that $x_{i}$ is not in the closure of $S \sim\left\{x_{1}, \cdots, x_{\jmath}\right\}$. Therefore $x_{\imath}$ has a neighborhood containing no points of $S \sim\left\{x_{1}, \cdots, x_{l}\right\}$, and sees no points of $K$ via $S$, which contradicts that $K\left(x_{i}\right) \neq \varnothing$. Therefore $S$ is starshaped.

On the other hand, suppose for some $x_{0} \in S, K\left(x_{0}\right)=\varnothing$. Then $x_{0}$ is the only point of $S$ with empty visibility set, and $\left(S \sim\left\{x_{0}\right\}, K\right)$ is of Helly type $(2 k+1,2 k+1)$. By Helly's theorem, the collection $\{K(y): y \in S \sim$ $\left.\left\{x_{0}\right\}\right\}$ has a nonvoid intersection, so $S \sim\left\{x_{0}\right\}$ is starshaped. $S$ consists of the starshaped set $S \sim\left\{x_{0}\right\}$ and the point $\left\{x_{0}\right\}$. Closure of $S$ implies that $x_{0}$ is isolated.

\section{REFERENCES}

1. M. Breen, An Example Concerning Unions of Two Starshaped Sets in the Plane, Israel J. of Math., 17 \#4 (1974), 347-349.

2. H. Hadwiger and H. DeBrunner, Über eine Variante zum Hellyschen Satz, Arch. Math., 8 (1957), 309-313.

3. F. A. Valentine, Convex Sets, McGraw-Hill, New York (1964).

Received July 2, 1975 and in revised form October 9, 1975. Partial support for the second author was provided by Pennsylvania State University through Grant PDE-OCC-EDUC-PROG IV \#3412.

Lehigh University, Bethlehem, Pennsylvania

AND

Pennsylvania State University, Worthington Scranton Campus 




\section{Pacific Journal of Mathematics}

\section{Vol. 62, No. $1 \quad$ January, 1976}

Mieczyslaw Altman, Contractor directions, directional contractors and

directional contractions for solving equations . .................. 1

Michael Peter Anderson, Subgroups of finite index in profinite groups .........

Zvi Arad, Abelian and nilpotent subgroups of maximal order of groups of odd order

John David Baildon and Ruth Silverman, On starshaped sets and Helly-type theorems ..........................................

John W. Baker and R. C. Lacher, Some mappings which do not admit an

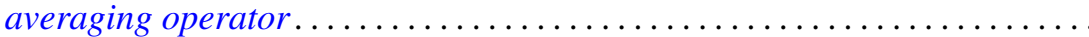

Joseph Barback, Composite numbers and prime regressive isols . . . . . . . . . .

David M. Boyd, Composition operators on $H^{p}(A) \ldots \ldots \ldots \ldots \ldots \ldots \ldots$

Maurice Chacron, Co-radical extension of PI rings . . . . . . . . . . . . .

Fred D. Crary, Some new engulfing theorems . . . . . . . . . . . . . . .

Victor Dannon and Dany Leviatan, A representation theorem for convolution transform with determining function in $L^{p} \ldots \ldots \ldots \ldots \ldots \ldots \ldots \ldots \ldots \ldots \ldots \ldots \ldots \ldots$

Mahlon M. Day, Lumpy subsets in left-amenable locally compact

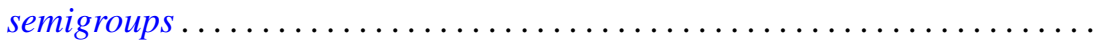

Michael A. Gauger, Some remarks on the center of the universal enveloping algebra of a classical simple Lie algebra . .

David K. Haley, Equational compactness and compact topologies in rings

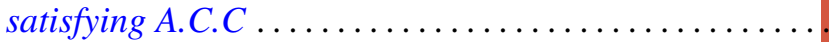

Raymond Heitmann, Generating ideals in Prüfer domains .

Gerald Norman Hile, Entire solutions of linear elliptic equations with

Laplacian principal part. .

Richard Oscar Hill, Moore-Postnikov towers for fibrations in which $\pi_{1}$ (fiber) is non-abelian

John Rast Hubbard, Approximation of compact homogeneous maps . .

Russell L. Merris, Relations among generalized matrix functions . .

V. S. Ramamurthi and Edgar Andrews Rutter, On cotorsion radicals ...

Ralph Tyrrell Rockafellar and Roger Jean-Baptiste Robert Wets, Stochastic

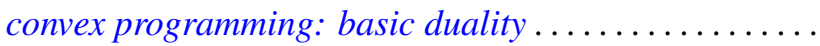

Alban J. Roques, Local evolution systems in general Banach spaces ..

I. Bert Russak, An indirect sufficiency proof for problems with bounded state variables.

Richard Alexander Sanerib, Jr., Ultrafilters and the basis property. .

H. A. Seid, The decomposition of multiplication operators on $L_{p}$-spaces . .

Franklin D. Tall, The density topology .................. 